\title{
Single-molecule kinetic energy of condensed normal deuterium
}

\author{
F. J. Mompeán, M. García-Hernández, and F. J. Bermejo \\ Instituto de Estructura de la Materia, Consejo Superior de Investigaciones Científicas, Serrano 123, E-28006 Madrid, Spain \\ S. M. Bennington \\ Isis Pulsed Neutron Facility, Rutherford Appleton Laboratory, Chilton, Didcot, Oxfordshire, OX11 OQX, United Kingdom
}

(Received 17 January 1996; revised manuscript received 18 March 1996)

\begin{abstract}
Inelastic scattering of 300-meV neutrons allows the study of the liquid ( $T=20$ and $30 \mathrm{~K}$, saturated vapor pressure) and solid $(T=4.2 \mathrm{~K}$, saturated vapor pressure $)$ phases of the normal deuterium mixture $\left(\frac{2}{3} o-\mathrm{D}_{2}+\frac{1}{3}\right.$ $\left.p-\mathrm{D}_{2}\right)$ in the region of momentum transfer where a single-molecule response is expected. The spectra are analyzed within the impulse approximation and assuming Gaussian momentum distributions for the translation of the molecules. For the solid, the estimated value of the single-molecule average kinetic energy does not compare unfavorably with those obtained scaling experimental results in parahydrogen solids. In the liquid state, substantial departures seem to exist from the classical liquid behavior, even if up to second-order quantum corrections are taken into account. [S0163-1829(96)01026-0]
\end{abstract}

\section{INTRODUCTION}

The solid phases of hydrogens attract attention given the unique combination of effects derived from the many mixed manifestations of their quantum and molecular characters. ${ }^{1,2}$ For low-pressure solids, several differences in the behavior of the $\mathrm{H}_{2}$ and $\mathrm{D}_{2}$ solids at temperatures below $1 \mathrm{~K}$ have recently been summarized and investigated further by $\mathrm{NMR}^{3}$ In the liquid state the peculiarities of the hydrogens are well reflected in the deviation of various thermodynamic and transport properties from the predictions of the principle of corresponding states on classical ${ }^{4}$ and quantum ${ }^{5}$ grounds. As is well known, provided that the appropriate kinematical restrictions are taken into account when analyzing the different contributions to the response functions, the experimental study of the dynamics of solids and of liquids far from the hydrodynamical regime is most conveniently made by neutron inelastic scattering. For the condensed phases of diatomics, and for $\mathrm{H}_{2}$ and $\mathrm{D}_{2}$ in particular, this point was dealt with extensively in the literature. ${ }^{6-8}$ In comparison to $\mathrm{H}_{2}$, the interpretation of the neutron inelastic double-differential cross sections at low momentum transfers is more fructiferous in the case of $\mathrm{D}_{2}$, since the smaller difference in the scattering lengths from the singlet and triplet compound nuclear states $\left(b^{(+)}=0.95 \times 10^{-12} \mathrm{~cm} b^{(-)}=0.10 \times 10^{-12} \mathrm{~cm}\right)$ results in a significant degree of coherent scattering, which allows the experimental observation of interference effects at low values of momentum transfer. In the liquid state, this fact has recently been exploited and inelastic scattering experiments on continuous thermal and cold neutron sources have recently been reported. ${ }^{9,10}$

In the present paper we report our measurements and analysis of the inelastic cross sections obtained from normal deuterium at an incident neutron energy $E_{i}=300 \mathrm{meV}$ for two temperatures $(T=20$ and $30 \mathrm{~K})$ under saturated vapor pressure in the liquid range and from a solid sample at $4.2 \mathrm{~K}$. A previous neutron inelastic study on liquid and solid normal and para- $\mathrm{H}_{2}$ has been reported ${ }^{11}$ in which the range of momentum transfer explored brings about a single-molecule re- sponse which can be described within the impulse approximation for molecules, and which is free of intermolecular collisional final state effects similar to those observed in the helium liquids at intermediate momentum transfer. ${ }^{12}$ More recently, Herwig et al. ${ }^{13}$ determined the density dependence of the single-molecule kinetic energy of solid para- $\mathrm{H}_{2}$, and found good agreement with predictions based on variational calculations. It seems natural to try to extend these studies to the case of $\mathrm{D}_{2}$ for two reasons. First, the theoretical predictions can be tested for the corresponding mass and zero-point vibrational amplitude effects once the experimental scalings are established. Second, in the case of $\mathrm{D}_{2}$ it is possible, given the slow rate for para-ortho conversion, to maintain for long measuring times quenched mixtures of para and ortho molecules without any significant changes in the composition. At low temperatures, virtually all the para- $\mathrm{D}_{2}$ molecules (one third of those in the sample) are in $J=1$ rotational states, and are the only contributors to the anisotropic part of the intermolecular potential. It is of interest to verify whether any experimental differences arise in the translational response to neutrons of the two populations in the sample in more advantageous conditions than in fast converting normal $\mathrm{H}_{2}$ mixtures.

Recent interest in the literature on neutron scattering by the hydrogens has focused on two aspects. Deep inelastic neutron-scattering studies, probing the single-atom response, from liquid and solid para- $\mathrm{H}_{2}$ (Ref. 14) and liquid (normal) $\mathrm{D}_{2}$ (Ref. 15) have been published, focusing their attention on the momentum distributions of the atoms in the condensed molecular system. At lower values of momentum transfer, single differential cross sections of liquid (normal) $\mathrm{D}_{2}$ have also been reported, analyzed within the static approximation $^{16}$ and related to results obtained by the pathintegral Monte Carlo simulation for the centers of mass. ${ }^{17}$ We believe our data provide information which will contribute to a complete picture of liquid deuterium as gained from these complementary techniques. Other spectroscopic techniques are being applied to the study of these systems: $\mathrm{Oka}^{18}$ recently reviewed high-resolution spectroscopy of solid 


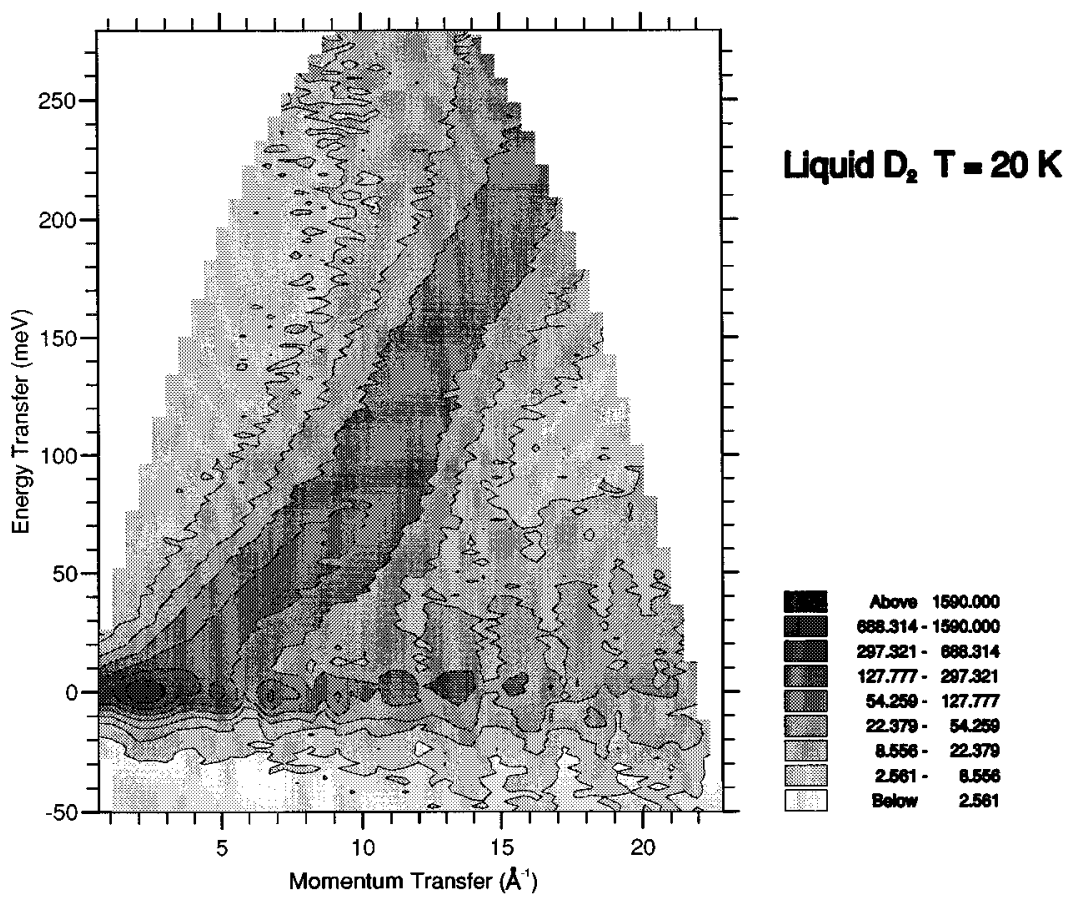

FIG. 1. Contour plot corresponding to $S(Q, E)$ of liquid normal $\mathrm{D}_{2}$ at $20 \mathrm{~K}$ under saturated vapor pressure before background subtraction and multiple-scattering correction.

para- $\mathrm{H}_{2}$, and the infrared spectra of vibrotational bands in liquid $\mathrm{H}_{2}$ and $\mathrm{D}_{2}$ have been reported. ${ }^{19}$

The rest of the paper is structured as follows: Sec. II contains details of the experimental setup and data correction procedures. Section III discusses the theoretical framework for the data analysis. Section IV summarizes the experimental results and presents details of the models used in the analysis. Section V includes a general discussion of the results. Finally, the conclusions and perspectives following our study are summarized in Sec. VI.

\section{EXPERIMENTAL DETAILS AND DATA CORRECTION}

The experiments were carried out at the direct-geometry chopper spectrometer MARI at the Isis Pulsed Neutron Source (Rutherford Appleton Laboratory, United Kingdom) using a neutron incident energy of $300 \mathrm{meV}$. Our choice of $300 \mathrm{meV}$ was set below the threshold for the excitation of the first intramolecular vibrational quantum $(371 \mathrm{meV}$ in the case of the $\mathrm{D}_{2}$ molecule). In this way, we fulfill the objectives of restricting the spectral composition of the molecular response to the lowest manifold of vibrotational states while measuring spectra with sufficient energy-transfer resolution as to render acceptable estimates of the intrinsic linewidths of the observed spectral features, and covering experimentally a sufficiently wide range of momentum transfers. The sample was kept in a specially designed aluminium container which consisted of two hollow concentric cylinders with their common axis perpendicular to the spectrometer scattering plane. The thickness of the resultant cylindrical shell was $0.5 \mathrm{~cm}$. The overall length of the cylinders was $5 \mathrm{~cm}$, but a set of cadmium rings was inserted defining small sections 1 $\mathrm{cm}$ in height in order to further reduce multiple scattering. The sample container was attached to a gas handling system and placed in a cryostat with temperature sensors and heating elements attached. High-purity commercial $\mathrm{D}_{2}$ gas was then condensed while the ensemble was kept at $20 \mathrm{~K}$. During this process the normal deuterium mixture corresponding to the room-temperature ortho-para equilibrium is quenched, and the room-temperature ratio is retained at the cryogenic temperature since, in the absence of catalyzers, the conversion process is very slow. ${ }^{20}$ Within each of the ortho and para populations a Boltzmann equilibrium is promptly established among rotational states. In all subsequent data analysis we shall assume that the normal mixture composition $\left(\frac{2}{3}\right.$ ortho- $\mathrm{D}_{2}, \frac{1}{3}$ para- $\mathrm{D}_{2}$ ) was retained at low temperatures throughout the whole duration of the experiment $(72 \mathrm{~h}$ ).

Data from the sample were collected at two temperatures within the liquid range ( 20 and $30 \mathrm{~K}$ ). After that, the sample temperature was cooled rapidly to $4.2 \mathrm{~K}$ in order to carry out the solid measurements. In all cases, the sample was kept under saturated vapor pressure. Given the steps followed in the preparation of the solid sample, and the sample holder geometry, it can be deemed to consist of highly textured hcp polycrystals. ${ }^{1,21}$ Background and calibration runs were taken at $20 \mathrm{~K}$.

The raw data were converted to dynamical structure factors as a function of momentum $(Q)$ and energy $(E)$ transfers, $S(Q, E)$, using standard procedures available on the instrument software package. Figure 1 shows the dynamical structure factor as a function of $Q$ and $E$ at $20 \mathrm{~K}$ before container subtraction. Since line-shape analysis is mandatory in this kind of study, it is necessary to account properly for the different contributions to the instrumental resolution as a function of both energy and momentum transfers. For this purpose, the vanadium sample calibration runs were examined for elastic peak widths. In this way, the dependence of $Q$ on the instrumental resolution was established. The results from a Monte Carlo estimation of the variation of the resolution function, in the Gaussian approximation, were used to account for the dependence with energy transfer.

An estimate of the multiple-scattering effects in our experiment was obtained by means of the Monte Carlo code MSCAT. $^{22}$ The code was run with scattering kernels appropri- 
ate for the range of $Q$ and $E$ accessible at this incident energy. The models and parameters involved were derived from preliminary analysis of the constant $Q$ spectra. When the total scattering simulated by the code was close to the experimental measurements and following interpolation to constant $Q$, ratios of single to multiple scattering were derived which were incorporated to the model fitting program described below. In all cases, the multiple-scattering contribution amounted to less than $10 \%$, and affected predominantly the higher values of energy transfer within each constant $Q$ spectrum.

\section{THEORETICAL FRAMEWORK}

In the solid phases at low pressure and in the liquid state, the relative weakness of the anisotropic part of the intermolecular potential allows for the consideration of the molecular rotational degree of freedom from a single-molecule perspective, as a first approximation. ${ }^{2}$ The double-differential cross section for neutron scattering by a diatomic molecular gas with explicit treatment of the intramolecular nuclear spin correlations and within the rigid-rotor approximation for molecular rotation has been derived a number of times in the literature for different neutron incident energy ranges. ${ }^{6,7}$ If the degree of mixing of rotational states introduced by anisotropy is not high and nuclear-spin correlations between different molecules can be neglected, these formulas can be used in the analysis of the condensed phases. This viewpoint has been adopted previously in the analysis of scattering by the hydrogen solids and liquids. The resulting contributions to the dynamical structure factor can be obtained after convolution of the molecular contribution with $S_{s}(Q, E)$ and $S_{d}(Q, E)$, the space and time Fourier transforms of self and distinct van Hove correlation functions referred to the centers of mass of the molecules. The distinct correlation function will be accessed when the scattering amplitudes from the nuclei in different molecules can interfere. This situation arises after scattering events in which there is no change in the intramolecular (vibrotational and nuclear spin) quantum numbers, and accordingly the coherent scattering length appears in the relevant contributions to the cross sections. The information on the self-correlation functions is brought about in all cases. The precise choice of a model for $S_{s}(Q, E)$ and $S_{d}(Q, E)$ varies according to the particular range of $(Q, E)$ space which is being analyzed. As momentum transfer increases, the crossover to the domain of validity of the incoherent approximation can be effected. In these circumstances, the distinct correlation function can be approximated by the single-particle (molecule) correlation function. ${ }^{23}$ In this approximation, the resulting expressions for deuterium, the molecules being in the fundamental vibrational state, are [note that, as it is customary in the treatment of molecular liquids, the scattering functions $S_{\text {ortho }}(Q, E)$ and $S_{\text {para }}(Q, E)$ contain the scattering lengths]

$$
S(Q, E)=X_{\text {ortho }} S_{\text {ortho }}(Q, E)+\left(1-X_{\text {ortho }}\right) S_{\text {para }}(Q, E),
$$

where $X_{\text {ortho }}$ is the molar fraction of ortho molecules, and the contributions arising from ortho and para molecules are given by

$$
\begin{aligned}
S_{\text {ortho }}(Q, E)= & \sum_{J \text { even }} p_{J}\left\{\left(b_{\text {coh }}^{2}+\frac{5}{8} b_{\text {inc }}^{2}\right)\right. \\
& \times \sum_{J^{\prime} \text { even }} S_{s, \text { ortho }}\left(Q, E-E_{J J^{\prime}}\right) f\left(J, J^{\prime} ; Q, d\right) \\
& +\frac{3}{8} b_{\text {inc }}^{2} \sum_{J^{\prime} \text { odd }} S_{s, \text { ortho }}\left(Q, E-E_{J J^{\prime}}\right) \\
& \left.\times f\left(J, J^{\prime} ; Q, d\right)\right\}, \\
S_{\text {para }}(Q, E)= & \sum_{J \text { odd }} p_{J}\left\{\frac{3}{4} b_{\text {inc }}^{2} \sum_{J^{\prime} \text { even }} S_{s, \text { para }}\left(Q, E-E_{J J^{\prime}}\right)\right. \\
& \times f\left(J, J^{\prime} ; Q, d\right) \\
& +\left(b_{\text {coh }}^{2}+\frac{1}{4} b_{\text {inc }}^{2}\right) \sum_{J^{\prime} \text { odd }} S_{s, \text { para }}\left(Q, E-E_{J J^{\prime}}\right) \\
& \left.\times f\left(J, J^{\prime} ; Q, d\right)\right\}, \\
& \times \sum_{\lambda=\left|J^{\prime}-J\right|} C^{2}\left(J, J^{\prime}, \lambda ; 0,0\right) j_{\lambda}^{2}(Q d / 2) . \\
f\left(J, J^{\prime} ;\right. & Q, d)=4\left(2 J^{\prime}+1\right) \\
& J^{\prime}+J
\end{aligned}
$$

In the above expressions, we have allowed for different selfscattering functions for the ortho and para populations, $J$ and $J^{\prime}$ are the initial and final single-molecule rotational quantum numbers, $E_{J J^{\prime}}$ is the energy change in the corresponding transition, $p_{J}$ is the population factor for the molecular state with rotational quantum number $J$, calculated for the ortho and para species individually at the experimental temperature, $b_{\text {coh }}$ and $b_{\text {inc }}$ are the coherent and incoherent scattering lengths ( 0.67 and $0.40 \mathrm{fm}$, respectively), $C\left(J, J^{\prime}, \lambda ; 0,0\right)$ denotes a Clebsh-Gordan coefficient, $j_{\lambda}$ denotes a spherical Bessel function of order $\lambda$, and $d=0.74 \AA$ is the internuclear distance in the $\mathrm{D}_{2}$ molecule. In practice, all the sums over rotational states have been restricted to $J \leqslant 7$. The molecular rotational level energies were calculated allowing for quartic and sextic centrifugal distortion effects. This extension of the rigid-rotor model does not alter the rotational eigenfunctions, since the additional terms in the rotational hamiltonian do not mix components of the angular momentum operators. The values of the molecular constants, such as the rotational and centrifugal distortion constants in the ground vibrational state, were taken from the best gas phase estimates as collected in Ref. 1.

Our objective is to investigate whether for solid and liquid $\mathrm{D}_{2}$ in our experimental conditions, and for the range of incident neutron energies studied in this paper, the momentum transferred to the molecules by the neutron beam may be high enough to overcome the intermolecular forces and, in classical terms, cause the free recoil of the molecules. ${ }^{24}$ If the domain of application of this impulse approximation can be reached in our experiment for individual molecules (rather 
than for individual atoms) the position of the recoil-shifted rotational transitions will yield information on the effective mass of the recoiling units. In addition, line-shape analysis of the Doppler-broadened profiles should enable us to extract information on the width of the momentum distribution for the scattering units. Consequently, with these arguments we have adopted for the self-scattering functions a Gaussian form, ${ }^{25}$ and have explicitly accounted for the shift of the rotational transitions due to recoil

$$
S_{s}\left(Q, E-E_{J J^{\prime}}\right)=A(Q) \frac{1}{\sqrt{\pi W}} \exp \left(-\frac{\left(E-E_{J J^{\prime}}-E_{r}\right)^{2}}{W}\right)
$$

In the above expression, $E_{r}=\hbar^{2} Q^{2} / 2 M_{\text {eff }}$ is the recoil energy of a molecule of effective mass $M_{\text {eff }}$ after a scattering event with momentum transfer $Q . M_{\text {eff }}$ and $W$ are determined in the fitting procedure, together with $A(Q)$ : a global scaling factor which, in the absence of absolute calibrations, is needed to relate the experimental intensities to the predictions of the model. This scaling factor applies equally to all the transitions accounting for the spectra, in the same way as $M_{\text {eff }}$ and $W$, and does not alter the relative intensities for these transitions predicted by Eq. (3.2). Values of the effective mass $M_{\text {eff }}$ close to the free molecule mass (4 amu), will point out to single-molecule response and will enable the interpretation of the parameters of the model as kinetic magnitudes. In particular $E_{K}$, derived from the values of $M_{\text {eff }}$ and $W$ using $W=\frac{8}{3} E_{r} E_{K}$, can then be interpreted as the mean kinetic energy of the molecules which are considered to have initial states with a Gaussian distribution of centerof-mass momentum characterized by a width parameter $\sigma_{p}$ $=\sqrt{2 M_{\text {eff }} E_{K} / 3}$. As discussed by Sears, ${ }^{25}$ except in superfluid ${ }^{4} \mathrm{He}$, the Gaussian approximation is acceptable either on dynamical grounds (ideal gas, harmonic crystals) or as a consequence of the central limit theorem (quantum crystals).

\section{DATA ANALYSIS}

The spectra measured at the three experimental conditions show a remarkable similarity in their general features. These experimental $S(Q, E)$ surfaces were studied by performing cuts at constant $Q$ with integration steps of $0.2 \AA^{-1}$. At momentum-transfer values below $5 \AA^{-1}$, the experimental resolution does not allow a detailed study. In the region between 5 and $14 \AA^{-1}$, the spectra can be described as resulting from a shifting and broadening of transitions within a manifold of rotational levels. Eventually, at momentum transfers higher than $14 \AA^{-1}$, the main spectral features begin to fall outside the kinematically allowed region for the MARI spectrometer. The model expressions for $S(Q, E)$ were accordingly evaluated at constant $Q$ values appropriate for the integration step and convolved with an estimate, $R(Q, E)$, of the instrumental resolution in order to compare the prediction with the experimental data, after correction for multiplescattering effects. The determination of the model parameters was done by a nonlinear least-squares fitting procedure. It should be stressed that, in order to render a line-shape analysis practical, the full molecular contribution to $S(Q, E)$ should be evaluated. In this way it is ensured that the relative intensities of the molecular transitions falling in the range of
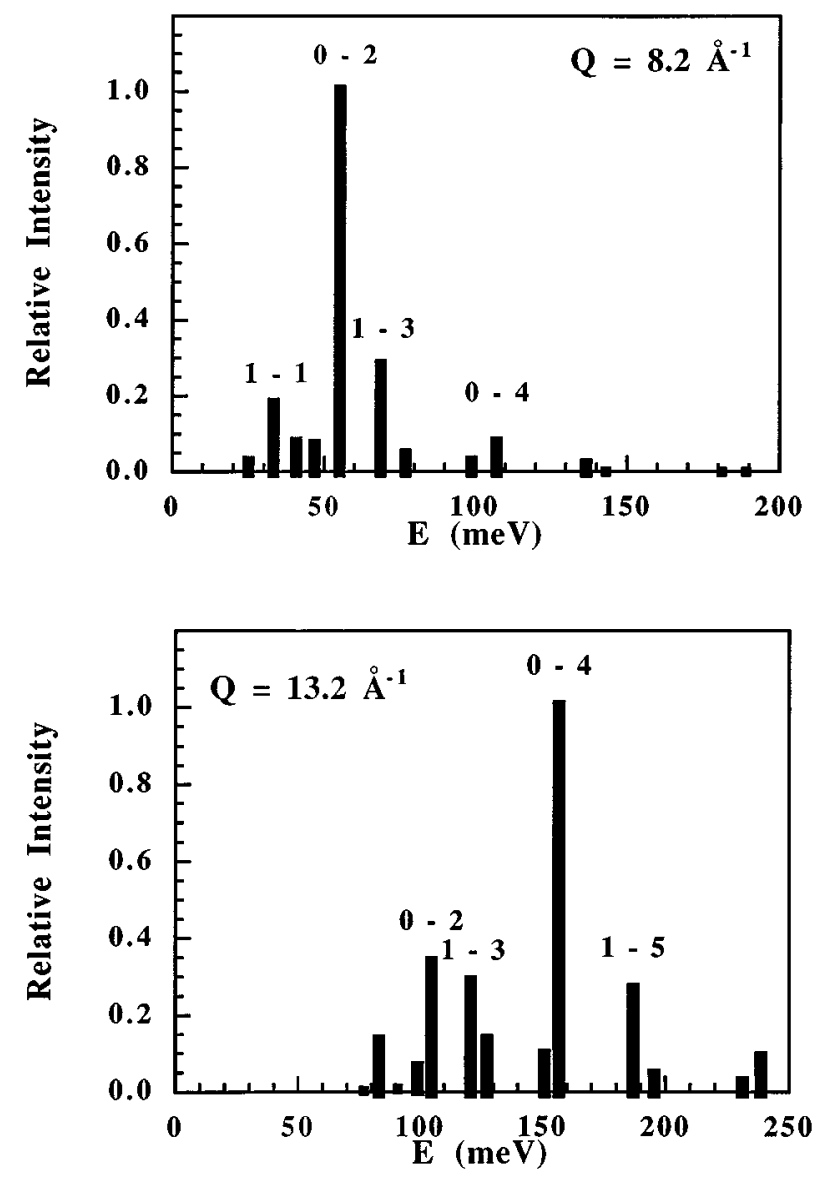

FIG. 2. Stick spectra for a normal $\mathrm{D}_{2}$ mixture with effective molecular mass of $4.1 \mathrm{amu}$ at $20 \mathrm{~K}$ corresponding to two values of momentum transfer $\left(Q=8.2 \AA^{-1}\right.$ and $\left.13.2 \AA^{-1}\right)$. The gas phase intensities for each contributing rotational transition have been normalized so that, in each spectrum, the more intense transition corresponds to unity. The numbers shown on top of the four more intense transitions identify the initial and final rotational quantum numbers $\left(J\right.$ and $\left.J^{\prime}\right)$.

$E$ being studied are being respected in the free-rotor limit. This fact is of particular practical importance in the analysis of condensed molecular deuterium as compared to the case of hydrogen since the small rotational constant of the former implies that the various rotational transitions that emerge to the $S(Q, E)$ surface at progressively higher values of $Q$ fall closer on the energy-transfer coordinate. Moreover, when dealing, as in the present case, with scattering from the normal liquid mixture, it should be borne in mind that transitions to the same final rotational state from the ortho and para populations will only differ by roughly $7.4 \mathrm{meV}$. The spectral complexity is illustrated by the stick spectra shown in Fig. 2 for two values of momentum transfer, assuming scattering from a hypothetical normal mixture deuterium gas $\left(M_{\text {eff }}=4.1 \mathrm{amu}\right)$ cooled to $20 \mathrm{~K}$.

The model specified by Eqs. (3.1)-(3.3) can be realized at different degrees of complexity. At the low temperatures of our experiment, the simplest one corresponds to setting $S_{s \text {,para }}(Q, E)$ equal to $S_{s, \text { ortho }}(Q, E)$. Careful line-shape analysis provides good estimates for the model parameters in spite of the spectral complexity of the normal mixture. Figures 3 
Solid $\mathrm{T}=4.2 \mathrm{~K}$
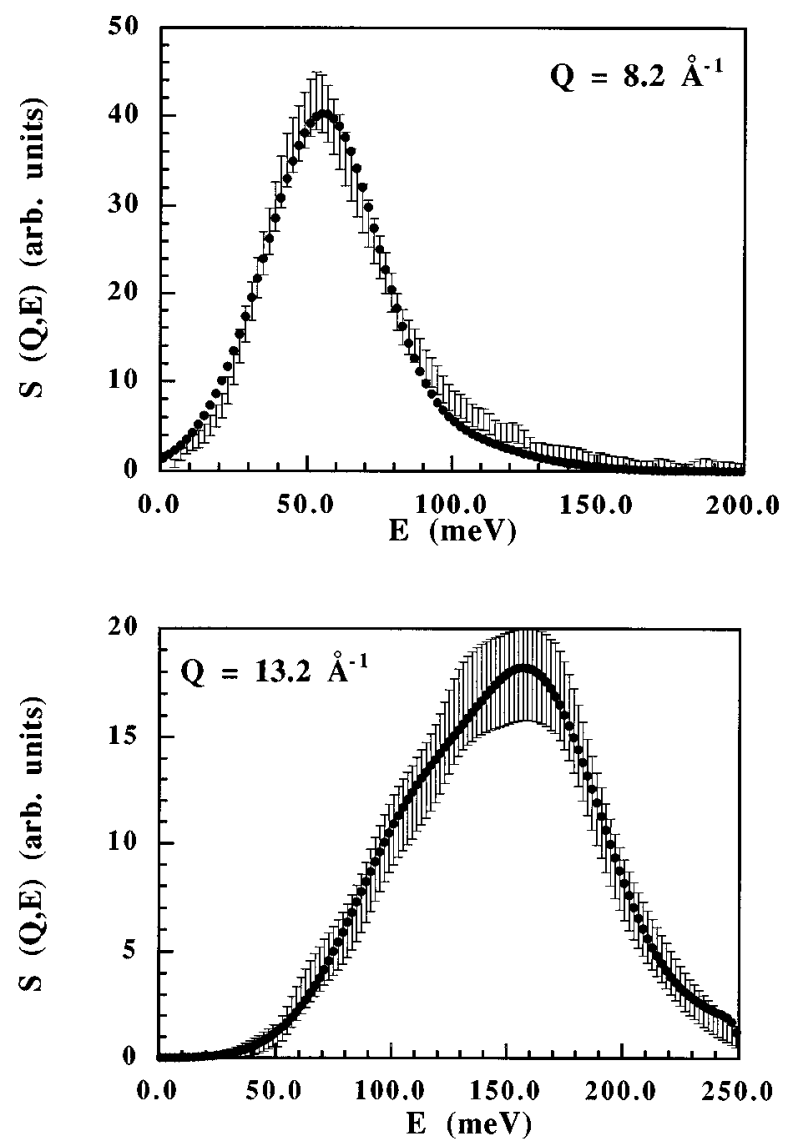

FIG. 3. Experimental data (centers of error bars) and best fit (black circles) for $S(Q, E)$ corresponding to the solid phase spectra at the two shown values of momentum transfer.

and 4 and 5 show representative spectra for two values of momentum transfer and the corresponding best fit to them using the model discussed above. Figure 6 shows a breakdown of the model (raw and convolved with the instrumental resolution) into the Gaussian components corresponding to the various contributing transitions for the liquid at $20 \mathrm{~K}$ and $Q=8.2 \AA^{-1}$. Reference to Fig. 2 is useful in identifying the series of transitions contributing in different regions of the spectra. It should be mentioned that in some cases, as shown in Fig. 4 for the liquid sample at $20 \mathrm{~K}$, deviations exist of the best estimates of the model function from the observed spectra. In the case of deviations noticed in the high-energy transfer tails of the spectra, it is possible to trace this mismatch to the approximate nature of the multiple-scattering corrections, but the ability of the gas phase intensities to account for the observed spectra seems to be limited in some other instances. Figures 7-9 contain the relevant fitting parameters as a function of momentum transfer. The global model scaling parameters $A(Q)$ are nearly constant over the range of fitted momentum transfers. This fact points out to the broad adequacy of the assumptions on the free-rotor limit and the normal ortho/para composition ratio. While the effective mass of the recoiling units seems to be fairly close to the free molecule value of $4 \mathrm{amu}$ in all cases, deviations from a linear dependence on $Q$ are observed for the width parameter $W$ at higher values of the momentum transfer. If, despite the anomalies mentioned above and discussed in Sec. V, we combine the
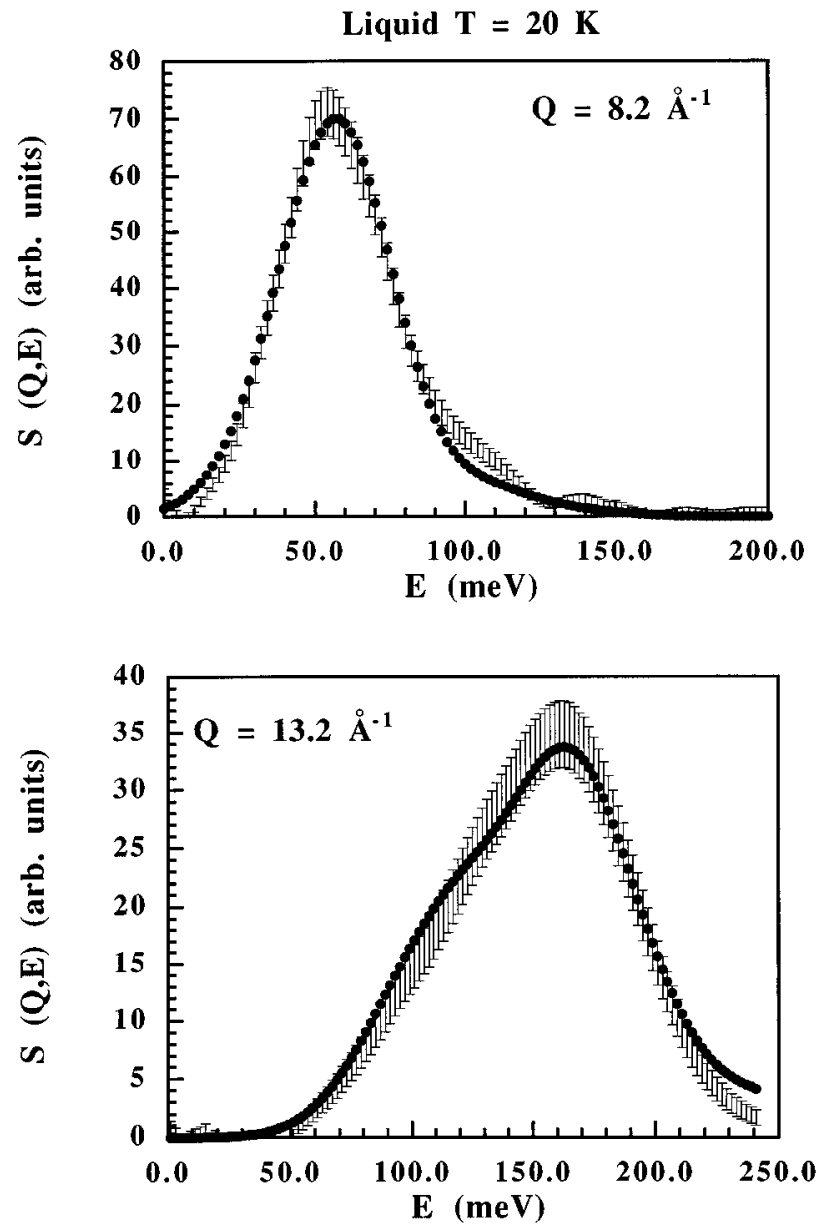

FIG. 4. As Fig. 3, but corresponding to the liquid at $20 \mathrm{~K}$ under saturated vapor pressure.

values, at each $Q$, of the effective mass and the width parameter, and subsequently average over the range of fitted momentum transfers, we can obtain estimates of values for the average kinetic energy per molecule, $E_{K}$. These are listed in Table I, together with a resume of the corresponding thermodynamic variables defining each measured state.

Two attempts were made to investigate further the problem of the departures from linearity in $Q$ of the experimental widths by adding complexity to the model. The first one consists of actually dispensing of the approximation of equal self-scattering functions for the ortho and para populations. The departures from linearity in $Q$ of the width corresponding to the ortho component continued to be present although the values were approximately $25 \%$, smaller and those corresponding to the para population showed a large degree of dispersion. It was not possible to correlate the dispersion of the latter to the absence in the spectra of rotational transitions initiated in the $J=1$ states, and we must conclude that the close spacing in the spectra of the transitions originating in ortho and para molecules and leading to the same rotational state precludes the accurate determination of individual line shape characteristics for the two populations. The second approach consisted in the use of the model proposed by Sears ${ }^{26}$ to actually account for departures at intermediate $Q$ values from the Gaussian line shape by means of the inclusion of a Gram-Charlier expansion in powers of the reciprocal momentum transfers. To this extent we supplemented 

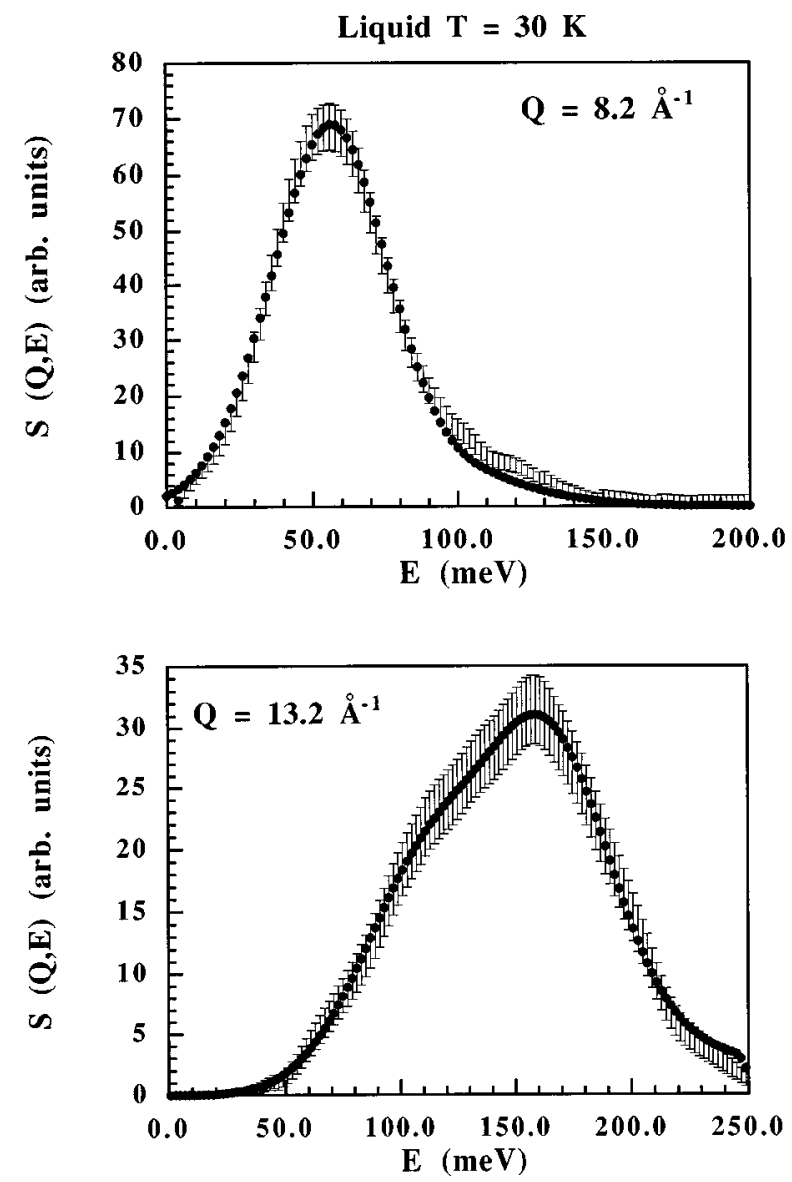

FIG. 5. As Fig. 3, but corresponding to the liquid at $30 \mathrm{~K}$ under saturated vapor pressure.

our Gaussian in Eq. (3.3) with an additional term depending on the fourth moment in $E$. The departures from linearity persisted for the term related to the second moment, and no significant improvement was found in the goodness of the fit parameter.

\section{DISCUSSION}

Our analysis has shown that the more general features of the observed inelastic scattering in a wide region of the $(Q, E)$ space seem to be correctly accounted for by our proposed model, although some anomalies are present. We focus our discussion first on those issues which we consider to have been firmly established, such as the existence of Gaussian translational momentum distributions in the three thermodynamical states explored whose widths follow a roughly linear dependence with momentum transfer. Finally we shall comment on various physical mechanisms that can be at the root of the observed deviations.

In the first place, it is interesting to verify if the magnitudes derived from our line-shape analysis result in estimates for the average kinetic translational energy which are close to the predictions based on the observations in similar systems $\left(\mathrm{H}_{2}\right)$ and common models. Our experimental values should be considered as averages over the two populations of ortho and para molecules present in the samples. In the solid phase, and assuming a Debye temperature $\theta_{D}$ of $114 \mathrm{~K},{ }^{27}$ for

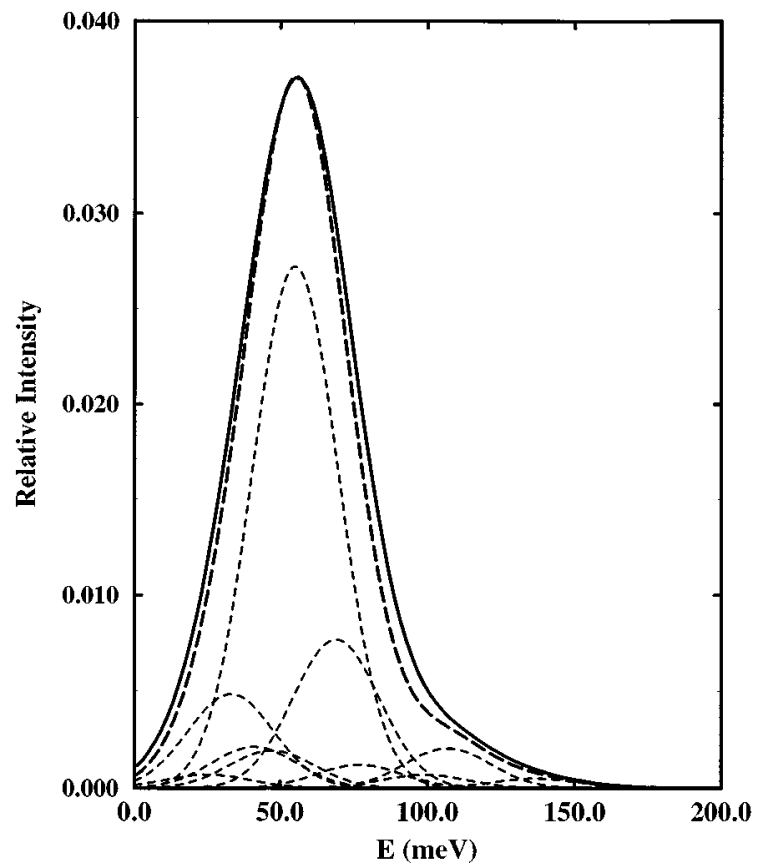

FIG. 6. Breakdown of the model best fit for the liquid at $20 \mathrm{~K}$ and $Q=8.2 \AA^{-1}$ into Gaussian components corresponding to individual rotational transitions. The short-dashed lines correspond to the individual contributions; the long-dashed line to the sum of the individual contributions and the solid line to the convolution of the latter with the experimental resolution. Except for the use of a relative intensity scale, established by requiring that the integrated area of the individual Gaussians should correspond to the relative intensity shown in Fig. 2, the solid line coincides with the best fit shown in Fig. 4.

a harmonic crystal at temperatures much lower than $\theta_{D}$ we would expect an average kinetic energy of $64 \mathrm{~K}$. Our experimental average for this latter magnitude is $68.5 \pm 11 \mathrm{~K}$, which is not in strong disagreement with an estimate of $72 \mathrm{~K}$ based on values of Herwig et al. ${ }^{13}$ for the corresponding

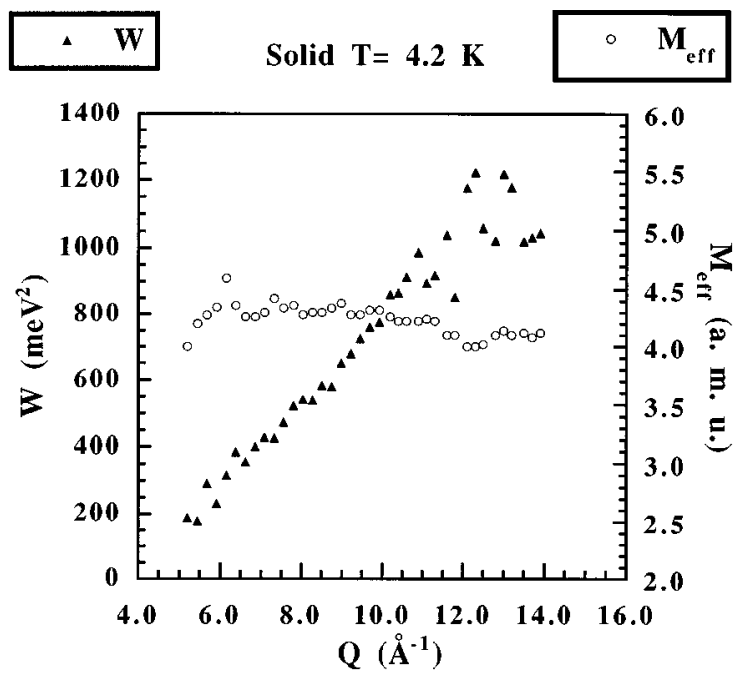

FIG. 7. Best-fit parameters as a function of momentum transfer for the solid sample at $4.2 \mathrm{~K}$ : left vertical axis shows values of width parameter $W$ (black triangles), and right vertical axes shows values of effective mass $M_{\text {eff }}$ (hollow circles). 


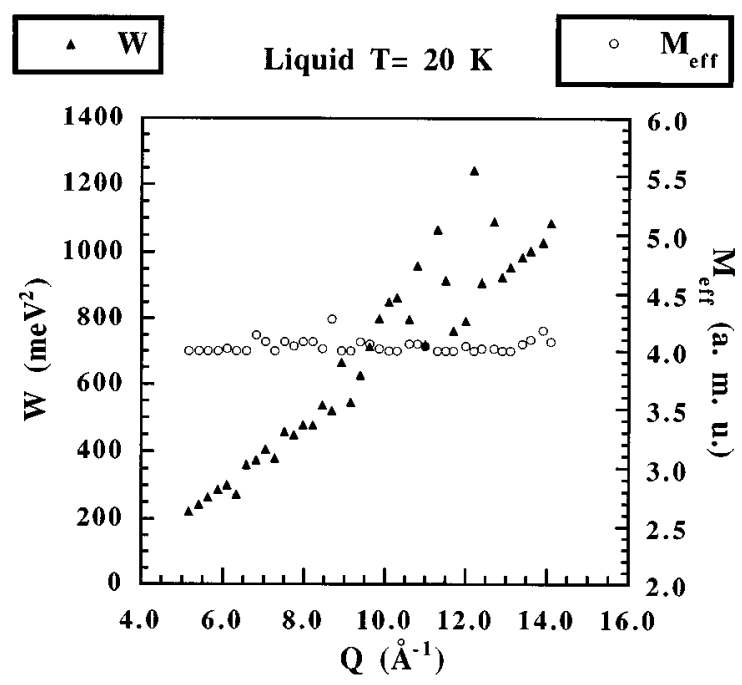

FIG. 8. As Fig. 6, but corresponding to liquid at $20 \mathrm{~K}$ under saturated vapor pressure.

magnitude in zero-pressure $p-\mathrm{H}_{2}$ at $4.2 \mathrm{~K}$, once the differences in mass and zero-point vibrational amplitude are taken into account. In the liquid state, the value of $E_{K}$ is found to increase with temperature (and therefore to decrease with increasing molar volume under saturated vapour pressure). An ideal gas scaling would predict the kinetic energy at $20 \mathrm{~K}$ to be 0.667 times the corresponding value at $30 \mathrm{~K}$. As can be expected, the experimental ratio of 0.93 deviates from this limit. Our data can be further compared with those obtained from the classical (atomic) liquid scaling with first- and second-order quantum corrections. To do this, we have tried to calculate the values of the characteristic temperature $\theta=\left(\hbar^{2}\left\langle\nabla_{r}^{2} \Phi\right\rangle / 3 M k_{B}^{2}\right)^{1 / 2}$, where $\left\langle\nabla_{r}^{2} \Phi\right\rangle$ is the classical average of the Laplacian of the intermolecular potential, which would account for the observed kinetic energies in the two liquid states if the expansion given by Sears ${ }^{25}$ for the effective temperature $T_{0}$ corresponding to $\frac{2}{3} E_{K}$ were valid for our system:

$$
T_{0}=T\left\{1+\frac{1}{12}\left(\frac{\theta}{T}\right)^{2}-\frac{1}{240}\left(\frac{\theta}{T}\right)^{4}+\cdots\right\} .
$$

However, the observed averages for 20 and $30 \mathrm{~K}$ cannot be accounted for by the sole inclusion of the first- and secondorder quantum corrections explicit in (5.1). This inadequacy is more pronounced for the lower temperature. We must conclude the presence in the liquid phase of normal deuterium of fairly significant quantum effects in the translation of the molecules in the liquid phase.

TABLE I. Values of the average single-molecule kinetic energy as a function of the thermodynamic state (all points under saturated vapor pressure).

\begin{tabular}{ccc}
\hline \hline$T(\mathrm{~K})$ & $E_{K}(\mathrm{meV})$ & Molar volume $\left(\mathrm{cm}^{3} / \mathrm{mole}\right)$ \\
\hline 4.2 & $5.9 \pm 1.0$ & 19.9 \\
20 & $5.2 \pm 0.8$ & 23.5 \\
30 & $5.6 \pm 0.8$ & 28.0 \\
\hline \hline
\end{tabular}

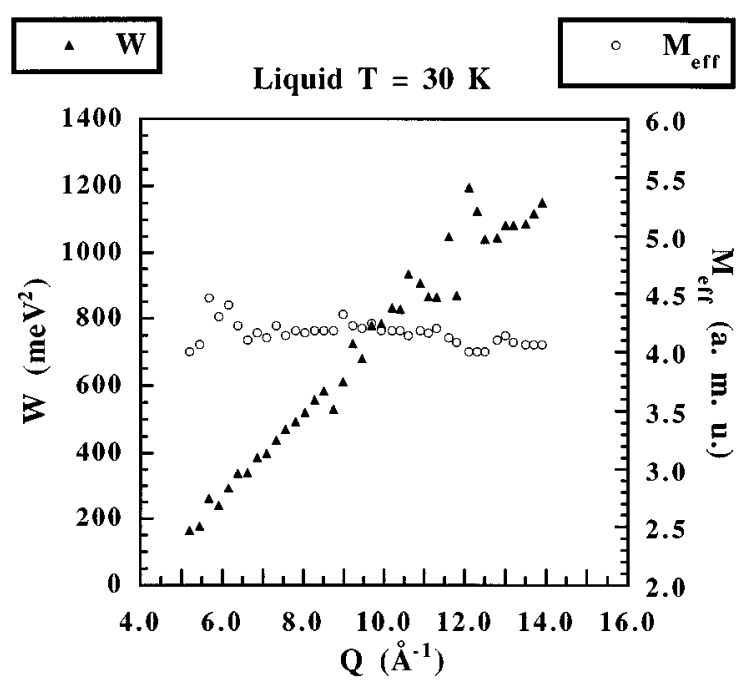

FIG. 9. As Fig. 6, but corresponding to liquid at $30 \mathrm{~K}$ under saturated vapor pressure.

The fact that the deviations in the linearity in $Q$ of the width parameter (at the higher values of momentum transfer) are present in the three measured thermodynamic states of condensed deuterium suggest that, to a good extent, the intramolecular degrees of freedom play a role in the underlying mechanism. The two open possibilities are either differences in the momentum distribution for ortho- and para- $\mathrm{D}_{2}$ molecules or final-state effects. We have already exposed our failure to extract individual momentum distribution parameters for the two populations in our sample. Here we shall elaborate qualitatively on the nature of the final-state effects that can be expected to take place in these particular molecular systems. In this case we expect contributions arising from significant differences in the intermolecular potential experienced by the molecules when placed at different translational and rotational final states by high values of energy and momentum transfer from the neutron beam, and effects related to the broadening of these final states. At our experimental values of momentum transfer, the recoiling molecule is left not only with a translational energy much greater than that of its neighbors (given the small probability of neutron scattering) but also in a highly excited rotational state. The anistropic contributions to the intermolecular potential are different for molecules in different rotational excited states, and certainly different from those experienced by those in $J=0$ (ortho) and $J=1$ (para) initial states. Of course, at least in the solid phase and for the case of purely rotational excitation, it is well known that anisotropic terms in the intermolecular hamiltonian give rise to energy bands. In such a periodic medium, these bands correspond to global states in which some of the individual molecules are rotationally excited. ${ }^{2}$ The density of states for some of these bands has been calculated, and the corresponding energy spreads can be of the order of a few $\mathrm{meV}$, and they have been reported to roughly account for infrared and Raman spectra in solid para- $\mathrm{H}_{2} \cdot{ }^{28} \mathrm{In}$ a classical picture, broadening of final states can be understood as follows: the excited molecule will experience a series of collisions in which translational and rotational energy will be exchanged. The values of the energies needed for the various rotational exchanges are by no means small in com- 
parison with the translational energies, and it is conceivable that a certain amount of momentum needs be transferred to the molecules before some of the rotational deexcitation channels are actually available. As a result, at larger momentum transfers the number of rotational deexcitation channels is higher. This conjecture may explain the observed behavior, since the lifetime of the final states of the molecules will differ substantially depending on whether or not they are placed in states from which it is possible to promptly decay to the fundamental state.

\section{CONCLUSIONS}

Neutron inelastic scattering at intermediate momentum transfers has proven useful in the study of single-molecule properties in condensed normal deuterium. In the solid phase at $4.2 \mathrm{~K}$, we find a good agreement with published experimental and theoretical results for para- $\mathrm{H}_{2}$, after accounting for mass and zero-point collective vibrations displacement effects. In going from the solid phase at $4.2 \mathrm{~K}$ to the liquid near the triple point, we observe a decrease in the kinetic energy which is qualitatively compatible with the corre- sponding increase in the molar volume. In the liquid phase, the single-molecule kinetic energy and consequently the momentum distribution for the higher measured temperature (30 $\mathrm{K}$ ) is greater than the one corresponding to the sample near the triple point $(20 \mathrm{~K})$. The observed values obtained for these quantities at the two temperatures under saturated vapor pressure suggest a strong deviation from the classical liquid behavior, even with allowance for first- and secondorder quantum corrections. Finally we point out that a quantitative assessment of the final-state effects present in these experiments may constitute a valuable tool for the study of the mechanisms for intermolecular translational to rotational energy transfer in these quantum systems.

\section{ACKNOWLEDGMENTS}

The authors are grateful to Ian Bailey and the rest of Sample Environment Group of the Isis Facilty for their help during the course of this experiment. This research has been made possible in part by the Spanish DGICYT through Grant No. PB92-0015.
${ }^{1}$ I. F. Silvera, Rev. Mod. Phys. 52, 393 (1980).

${ }^{2}$ J. Van Kranendonk, Solid Hydrogen: Theory of the Properties of Solid $\mathrm{H}_{2}, \mathrm{HD}$ and $\mathrm{D}_{2}$ (Plenum, New York, 1983).

${ }^{3}$ A. B. Harris, H. Meyer, and X. Qin, Phys. Rev. B 49, 3844 (1994).

${ }^{4}$ J. Rouch, J. P. Boon, and P. A. Fleury, Physica A 88, 347 (1977).

${ }^{5}$ L. H. Nosanow, J. Phys. (Paris) Colloq. 41, C-1 (1980).

${ }^{6} \mathrm{G}$. Sarma, Proceedings of the International Atomic Energy Agency Symposium on Inelastic Scattering of Neutrons in Liquids and Solids, Vienna, 1960 (International Atomic Energy Agency, Vienna, 1961).

${ }^{7}$ J. A. Young and J. U. Koppel, Phys. Rev. 3, A603 (1964).

${ }^{8}$ H. Stein, H. Stiller, and R. Stockmeyer, J. Chem. Phys. 57, 1726 (1972).

${ }^{9}$ F. J. Bermejo, F. J. Mompean, M. García-Hernández, J. L. Martinez, D. Martin-Marero, A. Chahid, G. Senger, and M. L. Ristig, Phys. Rev. B 47, 15097 (1993).

${ }^{10}$ F. J. Mompean, F. J. Bermejo, M. García-Hernández, B. Fåk, J. L. Martinez, G. Senger, and M. L. Ristig, J. Phys. Condens. Matter 5, 5743 (1993).

${ }^{11}$ W. Langel, D. L. Price, R. O. Simmons, and P. E. Sokol, Phys. Rev. B 38, 11275 (1988).

${ }^{12}$ H. R. Glyde, Excitations in Liquid and Solid Helium (Oxford University Press, Oxford, 1994).

${ }^{13}$ K. W. Herwig, J. L. Gavilano, M. C. Schmidt, and R. O. Simmons, Phys. Rev. B 41, 96 (1990).
${ }^{14}$ J. Mayers, Phys. Rev. Lett. 71, 1553 (1993).

${ }^{15}$ C. Andreani, A. Filabozzi, and E. Pace, Phys. Rev. B 51, 8854 (1995).

${ }^{16}$ E. Guarini, F. Barocchi, R. Magli, U. Bafile, and M. C. Bellissent-Funel, J. Phys. Condens. Matter 7, 5777 (1995).

${ }^{17}$ M. Zoppi, U. Bafile, E. Guarini, F. Barocchi, R. Magli, and N. Neumann, Phys. Rev. Lett. 75, 1779 (1995).

${ }^{18}$ T. Oka, Ann. Rev. Phys. Chem. 44, 299 (1993).

${ }^{19}$ A. R. W. McKellar and M. J. Clouter, Can J. Phys. 72, 51 (1994)

${ }^{20}$ H. M. Roder, G. E. Childs, R. D. McCarty, and P. E. Angerhofer, Survey of the Properties of the Hydrogen Isotopes Below Their Critical Temperatures, NBS Technical Note 641 (US Department of Commerce, Washington, 1973).

${ }^{21}$ R. L. Mills, J. L. Yarnell and A. F. Schuch, Proceedings of the Low Temperature Physics Conference 13, Boulder (Plenum, New York, 1973), Vol. II, p. 203.

${ }^{22}$ J. R. D. Copley, P. Verkerk, A. A. van Well, and H. Fredrikze, Comput. Phys. Comm., 40, 337 (1986).

${ }^{23}$ V. F. Sears, Phys. Rev. A 5, 452 (1972).

${ }^{24}$ J. M. F. Gunn, C. Andreani, and J. Mayers, J. Phys. C 19, 1835 (1986).

${ }^{25}$ V. F. Sears, Can. J. Phys. 63, 68 (1985).

${ }^{26}$ V. F. Sears, Phys. Rev. A 7, 340 (1973).

${ }^{27}$ M. Nielsen, Phys. Rev. B 7, 1626 (1973)

${ }^{28}$ S. K. Bose and J. D. Poll, Can. J. Phys. 68, 159 (1990). 